\title{
A influência do PET-Saúde na formação do estudante de Fisioterapia: relato de experiência
}

\author{
The influence of PET-Saúde in the formation of the Physiotherapy student: an experience report
}

\author{
Juliana de Oliveira Souzaa, Bruno Castro de Oliveirab \\ a Fisioterapeuta. Ex-bolsista do Programa de Educação pelo Trabalho para a Saúde (PET-Saúde), Centro Universitário de Barra Mansa (UBM), Barra Mansa, RJ, Brasil. \\ b Enfermeiro. Especialista em Saúde da Família, Centro Universitário Oswaldo Aranha (UniFOA) e Gestão Pública Municipal, Universidade Federal Fluminense (UFF), \\ Volta Redonda, RJ, Brasil. Ex-preceptor do PET-Saúde (UBM).
}

RESUMO Objetivo: Relatar a experiência vivenciada por uma estudante do curso de Fisioterapia do Centro Universitário de Barra Mansa (UBM) durante sua participação no Programa de Educação pelo Trabalho para a Saúde (PET-Saúde) e a influência do programa sobre sua formação profissional.

Descrição da experiência: A experiência foi vivenciada em uma Unidade Básica de Saúde da Família (UBSF) de um município do estado do Rio de Janeiro, onde foram realizadas atividades como projetos de pesquisa, visitas domiciliares aos usuários, ações voltadas à promoção da saúde e prevenção de agravos de doenças crônicas não transmissíveis, eventos para a comunidade e campanhas nas UBSF. Além das atividades na UBSF, ocorreram reuniões mensais com os coordenadores, tutores, preceptores e estudantes na instituição de ensino. Durante esses encontros eles compartilharam experiências, avaliaram os trabalhos realizados e discutiram sobre a necessidade de novas atuações.

Conclusão: Considera-se que o PET-Saúde contribuiu para a formação profissional da estudante de Fisioterapia, pautada no posicionamento crítico e reflexivo. A participação no programa a permitiu conhecer o ambiente de trabalho do SUS, adquirir novos conhecimentos, produzir trabalhos científicos, interagir com a comunidade na qual esteve inserida e com os profissionais que atuam na saúde do município, valorizar o trabalho em equipe e, sobretudo, reconhecer a importância da inserção do fisioterapeuta na atenção básica a saúde.

Palavras-chave: sistema único de saúde; atenção primária à saúde; estratégia saúde da família; fisioterapia.

\section{ABSTRACT}

Objective: To report the experience lived by a student of the Physiotherapy course in the University Center of Barra Mansa (UBM) during their participation in the Education Program for Working for Health (PET-Saúde) and the influence of the program on their professional training.

Description of experience: Experience occurred in a Basic Family Health Unit (UBSF) of a municipality in the state of Rio de Janeiro, where activities such as research projects, home visits to users, actions directed to health, promotion and prevention of aggravation of chronic diseases, events for the community and campaigns in the UBSF were performed. Besides the activities in UBSF, there were monthly meetings with the coordinations, tutors, preceptors and students of the educational institution. During these meetings they shared experiences, evaluated the work done and discussed the need for further actions.

Conclusion: It is considered that the PET-Saúde contributed to the vocational training of the physiotherapy student, based on critical and reflexive positioning. Participation in the program allowed her to meet the SUS workplace, acquiring new knowledge, producing scientific papers, interacting with the community in which it was inserted and the professionals working in municipal heath, valorizing teamwork and, most importantly, recognizing the importance of integrating the physiotherapist in the basic health care.

Keywords: unified health system; primary health care; family health strategy; physiotherapy. 


\section{INTRODUÇÃO}

O Sistema Único de Saúde (SUS) apresenta como porta de entrada a Atenção Básica à Saúde (ABS), nível em que aproximadamente $80 \%$ das necessidades de saúde de uma comunidade adstrita devem ser abordadas de maneira resolutiva ${ }^{1}$. Neste nível, a Estratégia Saúde da Família (ESF) auxilia na otimização e execução dos preceitos preconizados para a ABS. Uma de suas características é a preconização do trabalho em equipe a fim de garantir aos usuários uma assistência multiprofissional humanizada e individualizada que convertam atributos de cuidados indispensáveis para a preservação e promoção da saúde ${ }^{2,3}$.

O Programa de Educação pelo Trabalho para a Saúde (PET-Saúde) criado pelos Ministérios da Saúde e da Educação disponibiliza bolsas para tutores, preceptores e estudantes de graduação da área da saúde como incentivo ao estudo e a produção científica. Seu principal objetivo é fortalecer áreas estratégicas do SUS e promover maior aproximação e interação dos grupos de estudantes com a comunidade assistida pela $\mathrm{ESF}^{4,5}$.

Em parceria com as Secretarias Municipais de Saúde de dois municípios do estado do Rio de Janeiro, os cursos de Fisioterapia, Enfermagem e Farmácia do Centro Universitário de Barra Mansa (UBM), foram aprovados no PET-Saúde com um cronograma previamente elaborado para um período de 24 meses. Frente à proposta do Ministério da Saúde, os cursos do UBM e as secretarias de saúde elaboraram um projeto que contemplasse a reorientação da formação dos estudantes focada no conceito ampliado do processo saúde e doença, bem como nos seus fatores determinantes e condicionantes na perspectiva do SUS, com fomento a pesquisa em áreas específicas.

Acredita-se que o caráter interdisciplinar, um dos principais fundamentos do PET-Saúde, contribua positivamente no aprendizado profissional do estudante de Fisioterapia, visto que, segundo o Conselho Federal de Fisioterapia e Terapia Ocupacional (COFFITO), considera-se como atribuição especifica deste profissional participar de equipes multiprofissionais destinadas ao planejamento, implementação, controle e execução de projetos e programas de ações básicas de saúde ${ }^{6}$. Diante do exposto, este trabalho tem como objetivo relatar a experiência vivenciada por uma estudante do curso de Fisioterapia do UBM durante sua participação no PET-Saúde e a influência do programa sobre sua formação profissional.

\section{DESCRIÇÃO DA EXPERIÊNCIA}

Este trabalho consiste em um relato de experiência de caráter descritivo sobre as experiências vivenciadas, no ano de 2013, pela estudante de Fisioterapia em uma Unidade Básica de Saúde da Família (UBSF) de um município do estado do Rio de Janeiro, composta por duas equipes, que atendem uma população de aproximadamente 6.970 habitantes. As atividades na UBSF foram desenvolvidas durante um período de 6 meses, com carga horária de 12 horas semanais, cujos encontros aconteceram sob orientação do tutor e coordenação do preceptor. Tal experiência foi vivenciada em conformidade com os estudos teóricos e de campo, pautados no reconhecimento do território e levantamento de informações a cerca do eixo estruturante do PET-Saúde no município, no qual priorizava ações voltadas às doenças crônicas não transmissíveis.

A etapa inicial do programa se configurou por uma reunião na Secretaria Municipal de Saúde entre os coordenadores, tutores, preceptores e estudantes dos cursos de Fisioterapia, Enfermagem e Farmácia do UBM, aprovados para participarem do programa. Nesta reunião, a coordenação geral do PET-Saúde do UBM distribuiu os grupos de estudantes e preceptores por município.

A experiência na UBSF teve início na primeira reunião com as estudantes e as equipes, onde foi realizada uma análise dos relatórios gerenciais para reconhecimento do território, prevalência de doenças e óbitos, indicadores de saúde e observação do processo de trabalho das equipes. O grupo selecionado para atuar nesta UBSF era composto por três estudantes, sendo duas voluntárias dos cursos de Enfermagem e Farmácia e uma bolsista do curso de Fisioterapia, sendo coordenadas por um enfermeiro preceptor do programa. Após alguns meses de atividades, somente a estudante do curso de Fisioterapia prosseguiu com os trabalhos iniciados na UBSF. Sendo assim, durante as atividades propostas encontrou-se uma dificuldade em atingir o objetivo do programa, que consiste em integrar os estudantes de diferentes cursos e estimular o trabalho em equipe durante a constatação e resolução dos problemas.

Após a análise dos relatórios gerenciais, observou-se que na área de abrangência da UBSF havia um número significativo de usuários acamados e após este diagnóstico situacional, as ações foram direcionadas para a pesquisa. Foi elaborado um projeto de pesquisa e encaminhado para análise do Comitê de Ética em Pesquisa (CEP) do UBM com o objetivo de identificar informações sobre área, micro área, idade, sexo e patologias destes usuários e montar um banco de dados para analisar e traçar um perfil dessa população específica. O estudo foi desenvolvido com o apoio dos agentes comunitários de saúde (ACS) e a gerência da UBSF, que estiveram sempre integrados com as propostas do PET-Saúde.

A coleta de dados para a pesquisa foi realizada por meio dos prontuários dos usuários e de visitas domiciliares. A 
estudante realizou essas visitas na companhia de enfermeiros e/ou ACS, já que nesta UBSF não havia um Núcleo de Apoio a Saúde da Família (NASF) com a presença de fisioterapeuta. O município possui apenas um NASF e isso dificulta o acesso da população ao tratamento fisioterapêutico. A partir da constatação deste problema, a estudante e seu preceptor elaboraram um projeto para credenciamento e ampliação do NASF no município.

Além da coleta de dados para a pesquisa, durante as visitas domiciliares foram realizadas as seguintes atividades: avaliação fisioterapêutica do usuário acamado, esclarecimento sobre a doença apresentada pelo usuário; orientações quanto à prevenção de complicações, ao manuseio adequado do usuário, a possíveis adaptações ambientais no domicílio e a prevenção ou redução da sobrecarga do cuidador.

Durante sua participação no programa, a estudante ainda desenvolveu pesquisas relacionadas ao perfil de usuários portadores de feridas crônicas em membros inferiores e mapeamento territorial dos portadores de tuberculose no município. Além do desenvolvimento de pesquisas, a estudante participou de ações voltadas à promoção da saúde e prevenção de agravos de doenças crônicas não transmissíveis através das visitas domiciliares aos usuários, de eventos para a comunidade e campanhas nas UBSF.

Além das atividades desenvolvidas nos cenários de prática do PET-Saúde, ocorreram reuniões mensais com os coordenadores, tutores, preceptores e estudantes na instituição de ensino. Durante esses encontros eles compartilharam experiências, avaliaram os trabalhos realizados nos municípios e discutiram sobre a necessidade de novas atuações. Os relatos de experiência e os resultados de pesquisas foram divulgados em eventos promovidos pela coordenação geral do PET-Saúde do UBM e em congressos. Além disso, os dados coletados ficaram disponíveis para a instituição e foram fornecidos em forma de artigo científico para outros pesquisadores. Assim, analisando a evolução do programa, desde a sua implantação no âmbito acadêmico, é possível caracterizá-lo como uma valiosa estratégia de interação entre o ensino, o serviço e a comunidade.

\section{DISCUSSÃO}

A proposta de reunir uma equipe composta por estudantes de vários cursos em um único programa, como acontece no PET-Saúde, tem sido veiculada como uma estratégia para enfrentar o intenso processo de especialização na área da saúde, que no cenário atual, vem expandindo-se cada vez mais. Esse processo tende a aprofundar verticalmente o conhecimento acadêmico e a intervenção prática dos mesmos em aspectos individualizados das necessidades de saúde ${ }^{7}$.
A formação dos profissionais de saúde precisa ir além das práticas atuais e avançar no delineamento dos possíveis cenários sociais nos quais estarão inseridos os atuais estudantes, identificando as diferentes necessidades de saúde da população e ampliando o foco da formação profissional. Assim, é necessário um esforço de superação da visão tradicional da saúde como ausência de doença para uma visão que a considere como resultante das condições de vida da sociedade, das famílias e dos indivíduos, ou seja, como produto social ${ }^{8,9}$.

A percepção da importância da atuação do fisioterapeuta na ESF proporcionada pela participação no PET-Saúde permite ao estudante ter uma formação acadêmica diferenciada. O programa prepara o estudante para atuar em todos os níveis de atenção a saúde e o faz valorizar o trabalho em equipe e adquirir uma visão holística sobre o indivíduo em seu processo saúde-doença, operando de maneira preventiva e não somente restrita ao processo de reabilitação.

Apesar da atuação do fisioterapeuta na ESF ser fundamental, este profissional ainda não faz parte da equipe multidisciplinar que a compõe. Sendo assim, o Ministério da Saúde propõe a criação do NASF, através da Portaria no $154 / G M$, de 24 de Janeiro de 2008. Esta proposta busca o envolvimento de outros profissionais no apoio a ESF, visando à ampliação da rede de atenção básica à saúde na tentativa de melhorar a assistência ao indivíduo ${ }^{10}$.

A inserção do fisioterapeuta no NASF representa sua primeira aproximação com a atenção básica à saúde. Nos últimos anos, várias experiências de atuação da fisioterapia neste nível de atenção vêm sendo desenvolvidas. Essas experiências têm acontecido de forma pontual em alguns municípios brasileiros e nos espaços acadêmicos, em extensões universitárias e dentro da própria graduação em Fisioterapia como propõem as Diretrizes Curriculares Nacionais ${ }^{11}$.

Autores descrevem o objeto de ação do fisioterapeuta na ESF como "movimento humano visando à saúde funcional do indivíduo na promoção e recuperação da saúde, prevenção de doenças e agravos e na reabilitação" e o objetivo geral da sua atuação como o de "promover a qualidade de vida do indivíduo, tendo a integridade do movimento como essência e expressão desta"12. Durante as visitas domiciliares aos usuários e suas famílias observou-se a importância da atuação fisioterapêutica na atenção básica à saúde, com o intuito de melhorar a qualidade de vida desses usuários acamados que eram, em sua maioria, idosos portadores de doenças crônicas não transmissíveis.

O número de idosos com limitações e incapacidades geradas por essas doenças durante o envelhecimento é 
preocupante, devido à perspectiva do aumento desses casos, o que se reflete diretamente na saúde pública, no contexto social e político. Estudos mostram que a fisioterapia domiciliar oferece um resultado de 35\% de melhora considerável dos fatores relacionados ao quadro de restrição no leito ${ }^{13}$. Durante a experiência vivenciada, verificou-se que pela ausência do fisioterapeuta na UBSF, os usuários acamados encontravam-se dependentes de seus cuidadores, apresentando alterações cardiorrespiratórias e musculoesqueléticas importantes, além de úlceras de decúbito por conta da permanência prolongada no leito, evidenciando a importância do acompanhamento fisioterapêutico desses pacientes.

Além de atuar no ambiente domiciliar, estudantes de fisioterapia inseridos no PET-Saúde, também participam de atividades nas UBSF voltadas à promoção da saúde e prevenção de diversas doenças e seus agravos, contribuindo para o reconhecimento da rotina de um serviço público de atenção básica à saúde, com suas limitações e potencialidades ${ }^{14}$. No relato de experiência de Ferraz ele mostra que as pesquisas realizadas pelos estudantes participantes do programa também são importantes para a formação profissional dos mesmos. O autor afirma que o exercício da pesquisa permite aos acadêmicos e profissionais da saúde desenvolver atividades de caráter interdisciplinar, sendo um dispositivo para a troca de informações e saberes, além de fortalecer o vínculo e o acesso entre os serviços de saúde e a universidade ${ }^{15}$.

Diante do exposto, considera-se que o PET-Saúde contribuiu para a formação profissional da estudante de Fisioterapia, pautada no posicionamento crítico e reflexivo. A participação no programa a permitiu conhecer o ambiente de trabalho do SUS, adquirir novos conhecimentos, produzir trabalhos científicos, interagir com a comunidade na qual esteve inserida e com os profissionais que atuam na saúde do município, valorizar o trabalho em equipe e, sobretudo, reconhecer a importância da inserção do fisioterapeuta na atenção básica a saúde.

\section{REFERÊNCIAS}

1. Facchini LA, Piccini RX, Tomasi E, Thumé E, Silveira DS. Monitoramento e Avaliação do Projeto de Expansão e Consolidação da Saúde da Família (PROESF): Relatório Final do Estudo de Linha de Base do Lote 2 Sul. Pelotas: Universidade Federal de Pelotas; 2006. $316 \mathrm{p}$.
2. Sousa MF, Hamann EM. Programa Saúde da Família no Brasil: uma agenda incompleta? Cien saude colet 2009;14(5):1325-35. http:// dx.doi.org/10.1590/s1413-81232009000800002

3. Conill EM. Ensaio histórico-conceitual sobre a Atenção Primária à Saúde: desafios para a organização de serviços básicos e da Estratégia Saúde da Família em centros urbanos no Brasil. Cad Saúde Pública 2008;24(1):7-16. http://dx.doi.org/10.1590/S0102311X2008001300002

4. Santos BRL, Thiesen FV. O programa de educação pelo trabalho para a saúde fortalecendo a interdisciplinaridade na formação dos profissionais da área da saúde. Cienc Saude 2010;3(1):1 .

5. Brasil. Ministério da Saúde. PET-Saúde. 2014 dez [capturado 2014 dez 04]. Disponível em: http://portalsaude.saude.gov.br/

6. Brasil. Conselho Federal de Fisioterapia e Terapia Ocupacional. 2014 Dez [capturado 2014 dez 10]. Disponível em: http://www. coffito.org.br/site/

7. Araujo JS, Vidal GM, Brito FN, Alves CMR, Leite DKM, Dutra CDT, Pires CAA. Programa de educação pelo trabalho para a saúde: um relato de experiência. Cienc Saude 2012;5(1):54-8.

8. Brasil. Ministério da Saúde. Secretaria de Atenção à Saúde. Núcleo Técnico da Política Nacional de Humanização. Série B. Documento base para gestores e trabalhadores do SUS. Textos Básicos de Saúde. 3a ed. Brasília (DF): Ministério da Saúde; 2006. $52 \mathrm{p}$

9. Campos GWS. Humanização na saúde: um projeto em defesa da vida? Interface - Comunic Saúde Educ 2005;9(17):339-406. http:// dx.doi.org/10.1590/s1414-32832005000200016

10. Brasil. Ministério da Saúde. Núcleo de Apoio à Saúde da Família NASF. Diário Oficial da União. 2008 jan;1:47-9.

11. Brasil. Ministério da Educação. Diretrizes Curriculares Nacionais do Curso de Graduação em Fisioterapia. Diário Oficial da União. 2007 mar;1:11.

12. Pereira FWA, Mangueira JO, Monteiro MPA, Véras MMS, Lima VCS, Barrocas TCP, Lucena D. A inserção da fisioterapia na estratégia saúde da família em Sobral/CE. SANARE. [periódico online]. 2004 mar [capturado 2014 dez 18]; 5(1). Disponível em: http://sanare. emnuvens.com.br/sanare/article/view/127

13. Góis ALB, Veras RP. Fisioterapia domiciliar aplicada ao idoso. Rev bras geriatr gerontol 2006;9(2):300-8.

14. Mendes PMV, Silva EA, Santos LE, Carrias FMS, Prudêncio FA, Moura DR. A atuação fisioterapêutica no PET-Saúde UESPI "Atenção ao Idoso Acamado". Rev Eletrônica Gest Saúde 2014;9(1): 3151-8.

15. Ferraz L. O PET-Saúde e sua interlocução com o Pró-Saúde a partir da pesquisa: o relato dessa experiência. Rev bras educ med 2012;36(1):166-71. http://dx.doi.org/10.1590/S010055022012000200023 